\title{
Induce Hyperlipidemia in Rats Using High Fat Diet Investigating Blood Lipid and Histopathology
}

\author{
Karam I ${ }^{* 1}$, Ma N ${ }^{2}$, Yang Y-J ${ }^{2}$ and Li J- $\mathrm{Y}^{2}$ \\ ${ }^{1}$ Central Laboratory of Veterinary Research, Soba (Animal Resources Research Corporation ARRC) Sudan \\ ${ }^{2}$ Key Lab of New Animal Drug Project of Gansu Province; Key Lab of Veterinary Pharmaceutical \\ Development, Ministry of Agriculture; Lanzhou Institute of Husbandry and Pharmaceutical Science of \\ CAAS, Lanzhou, P.R. China
}

${ }^{*}$ Corresponding author: Karam I, Central Laboratory of Veterinary Research, Soba (Animal Resources Research Corporation ARRC) Sudan, Tel: 00249-912-624503, E-mail: isamkarm@yahoo.com

Citation: Karam I, Ma N, Yang Y-J, Li J-Y (2018) Induce Hyperlipidemia in Rats Using High Fat Diet Investigating Blood Lipid and Histopathology. J Hematol Blood Disord 4(1):104.

doi: $10.15744 / 2455-7641.4 .104$

Received Date: January 20, 2018 Accepted Date: August 15, 2018 Published Date: August 17, 2018

\begin{abstract}
The experiment was conducted to investigate the effect of induced hyperlipidemia in SD rats fed with high fat diet (HFD) for 7 weeks on blood lipid profile and possible pathological changes in liver, stomach, intestine, heart, spleen, lung and kidney. There were significant differences in most rats which induced hyperlipidemia throughout the experimental period with the blood lipid levels and histopathology.

Keywords: Rats; Hyperlipidemia; High Fat Diet; Histopathology
\end{abstract}

\section{Introduction}

Hyperlipidemia is modifiable risk factor for atherosclerosis and related cardiovascular diseases, including coronary heart disease, cerebral stroke, myocardial infarction and renal failure are becoming a major health problem in the world recently [1].

Hyperlipidemia is a heterogeneous group of disorders characterized by an excess of lipids in the blood stream, the term hyperlipidemia refers to increased concentrations of lipids (triglycerides, cholesterol, or both) in the blood [2-4]. These lipids include cholesterol, cholesterol esters, phospholipids, and triglycerides.

The term hyperlipoproteinemia refers to increased blood concentrations of lipoproteins, but it is often used interchangeably with the term hyperlipidemia. However, the term hyperlipoproteinemia should ideally be used only in cases where measurement of actual lipoproteins has been performed [4,5]. Lipids are transported in the blood as large lipoproteins, increased blood concentrations of triglycerides are referred to as hypertriglyceridemia, while increased blood concentrations of cholesterol are referred to as hypercholesterolemia.

Four main classes of lipids can be recognized from a metabolic stand point. These are free fatty acids, triacylglycerol, phospholipids, and cholesterol and its esters. The principle functions of lipids are to act as energy stores and to serve as important structural component of cells. To fulfill these functions, lipids have to be transported in plasma from one tissue to another, from the intestine or the liver to other tissues such as muscular or adipose tissue, or from the other tissues to the liver.

Lipids are water insoluble organic compounds, which are essential for many normal functions of living organisms: they are important components of cell membranes, they are used to store energy, and they play a significant role as enzyme co-factors, hormones, and intracellular messengers [6]. Of the many groups of lipids, three are most important from a clinical perspective: fatty acids, sterols (mainly cholesterol), and acylglycerols (mainly triglycerides) [6,7].

Fatty acids are relatively simple lipids and are also important components of many other lipids [6,7]. Cholesterol is the main sterol in animal tissues.

Dietary intake is the major source of cholesterol, but it can also be synthesized endogenously by the liver and other tissues. It plays a fundamental role in central metabolic pathways, such as bile acid metabolism and steroid hormone and vitamin D synthesis [6,7]. 
Triglycerides are the most common and efficient form of stored energy in mammal. They can be derived from both dietary sources and endogenous (hepatic) production [6,7].

Because lipids are water-insoluble molecules, they cannot be transported in aqueous solutions, such as plasma. For that reason, lipids are transported in plasma as macromolecular complexes known as lipoproteins [2,4,6-10].

Lipoproteins are spherical structures that consist of a hydrophobic core containing lipids (i.e. triglycerides and/or cholesterol esters), and an amphophilic (i.e. both hydrophobic and hydrophilic) outer layer of phospholipids, free cholesterol, and proteins that forms a protective envelope surrounding the lipid core $[4,6-8,10,11]$.

It is worth noting that free fatty acids are transported bound to albumin and do not require incorporation into lipoproteins for transport $[2,4,6,7,9,10]$. Plasma lipoproteins differ in their physical and chemical characteristics such as size, density, and composition. In this study induces hyperlipidemia in rats using dietary intake high fat diet for seven weeks.

Previous works have shown that feeding rats a high fat diet induces hepatic steatosis and liver damage, which are stages of the disease. Thus this study was designed to investigate the most histopathological changes which induces by feeding of high amounts of fats particularly cholesterol on the liver $[12,13]$.

\section{Materials and methods}

\section{Animals}

Twenty Sprague-Dawley (SD) rats SD male rats with clean grade (Certificate No.: SCXK (Gan) 2012-0075), aged 7 weeks and weighing 120 -130 g, were purchased from the animal breeding facilities of Gansu traditional Chinese medicine University (Lanzhou, China). They were housed in plastic Macrolon cages of appropriate size with stainless steel wire cover and chopped bedding. Light/ dark regime was $12 / 12 \mathrm{~h}$ and living temperature is $22 \pm 2{ }^{\circ} \mathrm{C}$ with relative humidity of $55 \pm 10 \%$. Standard compressed rat feed for group A and the other group B feed by high fat diet after two weeks of acclimatization, the Male SD rats was fed HFD (41.5\% lipids, $40.2 \%$ carbohydrates, and $18.3 \%$ proteins (kcal)). Weight, food intake, blood lipid parameters were measured during a 7 weeks diet course. Free access to either a standard rodent chow (12.3\% lipids, $63.3 \%$ carbohydrates, and $24.4 \%$ proteins (kcal)), or a high-fat diet, based on lard, swine oil, (derived from cod liver, HF-F).The food was supplied from Keao Xieli Co., Ltd (Beijing, China).

The study was performed in compliance with the Guidelines for the care and use of laboratory animals as described in the US National Institutes of Health. Animals were allowed a 2-week quarantine and acclimation period prior to start of the study.

\section{Feeding}

The rats were feeding with standard diet for two weeks before starting the experiment as acclimatization period for adaptation, then for group A as blank group continue for seven weeks as experimental period, for B group feeding with high fat diet for seven weeks.

\section{Study design}

After the administration period the rats divided in two in each group ten rats, blood lipids levels were measured, the examinations were carried out at the end of feeding administration; after the rats were feed with HFD food, the blood samples were collected from the rat tail, the rats will be fasting for 10-12 hours before collected blood samples. The serums were got through centrifuge for $15 \mathrm{mins}$ at the speed of $4000 \mathrm{~g}$ and stored at $-20^{\circ} \mathrm{C}$ till needed for lipid determination. The blood lipids level including TG, TC, HDL and LDL were measured on week 7 to make sure the success of hyperlipidemia animal model. Examinations of serum total lipids, was carried out at the end of experiment.

Blood sample was analyzed for hyperlipidemia indexes with a hematology using Erba XL-640 analyzer (German) was used to measure the blood lipid level. Moreover, an automatic biochemistry analyzer was used to examine the serum obtained from the blood sample for the content of TG, TC, LDL, and HDL.

\section{Histology}

After fixation (and subsequent weighing, vide supra), organs sampled for histological examination were dehydrated and paraffinized and embedded according to standard sampling and trimming procedures [14]. Sections of $4 \mu \mathrm{m}$ were stained with hematoxylin and eosin (HE) in an automated way. Microscopic observations were done by initial unblinded comparison of control and tested samples. Blind and/or semi-quantitative scoring was applied when changes were detected by the initial inspection.

\section{Statistics}

All data are expressed as mean \pm standard deviation (SD). The differences of ratios of indexes were analyzed using Dunette's test (SPSS 12.0 software, USA), and inter-group comparisons were made using the Multivariate of General Linear Model.P-values of $<0.05$ were considered statistically significant. 


\section{Results}

\section{Observations in animals' health}

No deaths were observed in any group over the administration periods. Compared with the control group, the test group exhibited no related changes in clinical signs such as external appearance, behavior, mental state, and daily activities. With regard to food consumption, all rats showed a significant increase in body weight (Table 1).

Table 1 showed increase in body weight.

\begin{tabular}{|c|c|c|c|c|c|c|c|}
\hline & Week 1 & Week 2 & Week 3 & Week 4 & Week 5 & Week 6 & Week 7 \\
\hline Model Group & 216.95 & 259.62 & 283.2 & 305.2 & 326.71 & 355.2 & 375.13 \\
\hline
\end{tabular}

"the weight per gram unit

Table 1: All rats in the tested groups had increased high body weights during experiment

\section{Blood lipid level}

The results of blood lipid level tests are given in Table 2. There was significant change in blood lipid level indexes which include TC, TG, LDL and HDL, compared with control group there was increase in TC, TG and LDL, while HDL was decrease.

\begin{tabular}{|c|c|c|c|c|}
\hline Week 7 & TG & HDL & LDL & TCH \\
\hline Blank group & 1.082143 & 0.384286 & 0.385714 & 1.73 \\
\hline Model group & $1.617488^{*}$ & $0.315255^{*}$ & $0.558319^{*}$ & $2.261139^{*}$ \\
\hline
\end{tabular}
Table 2: Blood lipid index experimental rats
Tabnificant difference from blank group

\section{Histopathology}

There were histopathological changes in organs from the animals in control group and tested group.

Liver cell were also observed there was infiltration of lipid (Figure 1). We did not find any other histopathological changes in any other organs assessed. The increased liver weights were associated with Centro lobular hypertrophy (Figure 1). In rats, there was a trend that the rats receiving high fat diet had a higher ratio of fat cells in their livers compared with control. Finally, an increased ratio of lipid hepatocytes was observed in exposed livers.
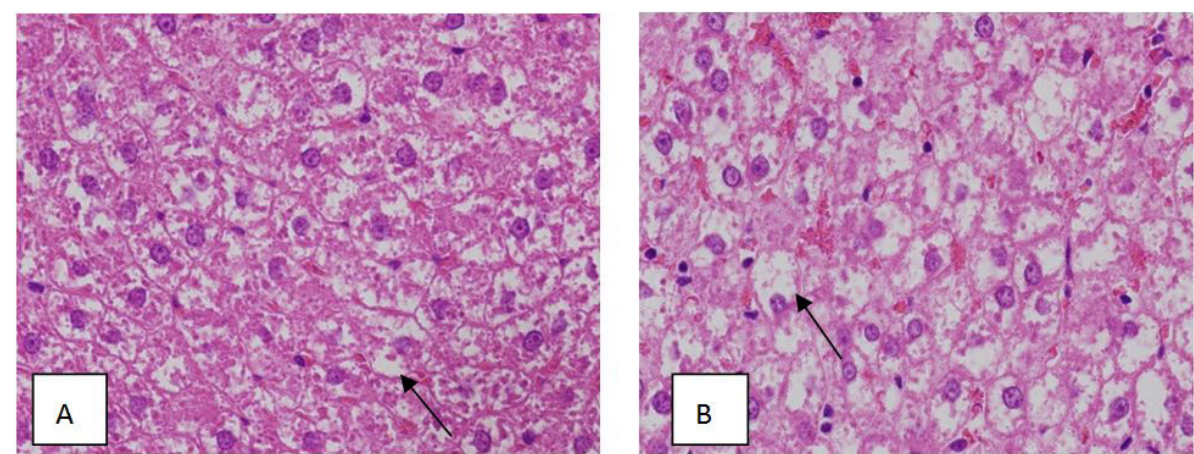

Figure 1: High power microphotographs of rat liver show lipid infiltration compare control B with tested group A

The results demonstrated that in the kidney lipid happened in the glomerulus of rats and the renal tubule interval of tested groups following high fat diet administration (Figure 2). No pathological changes such as degeneration and necrosis appeared in renal tubular epithelial cells in high fat diet groups, while more significant changes took place in tested groups.
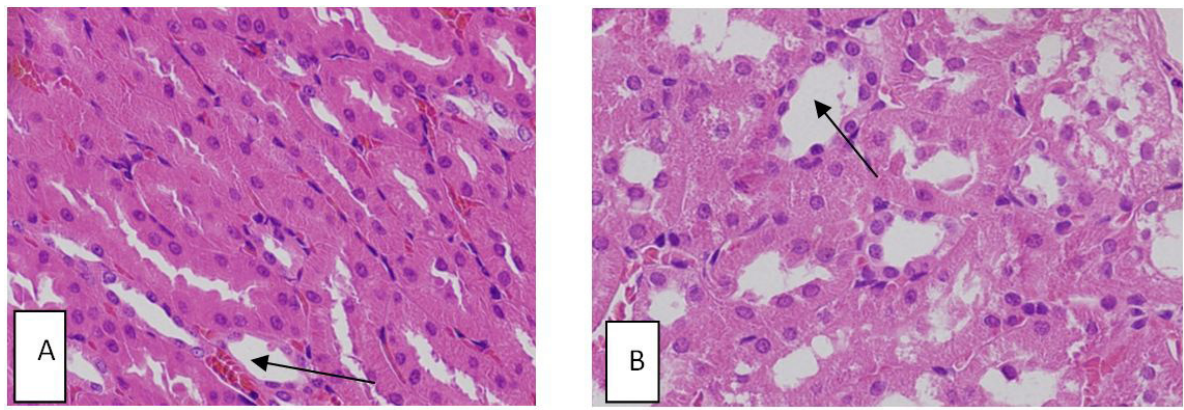

Figure 2: High power microphotographs of rat kidney show lipid infiltration compare control A with tested group B 
The stomach, duodenum and ileum showed an increased mucosa height after high fat diet exposure, this was mainly due to increased height of the villus (Figure 3).
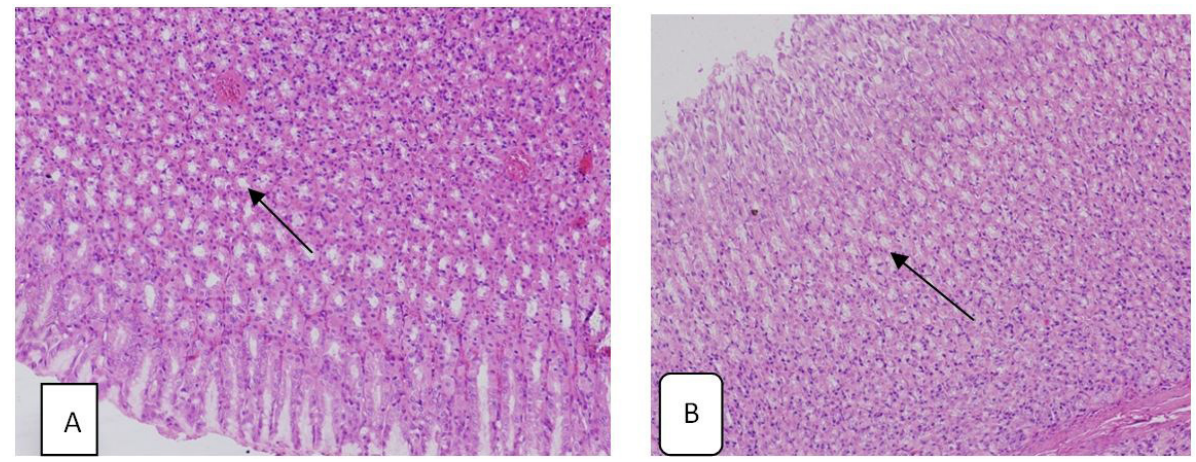

Figure 3: Low power microphotographs of rat stomach show lipid infiltration compare control A with tested group B

The results demonstrated that in the heart lipid happened in the cells (Figure 4) of rats and the muscle interval of tested groups following high fat diet administration.

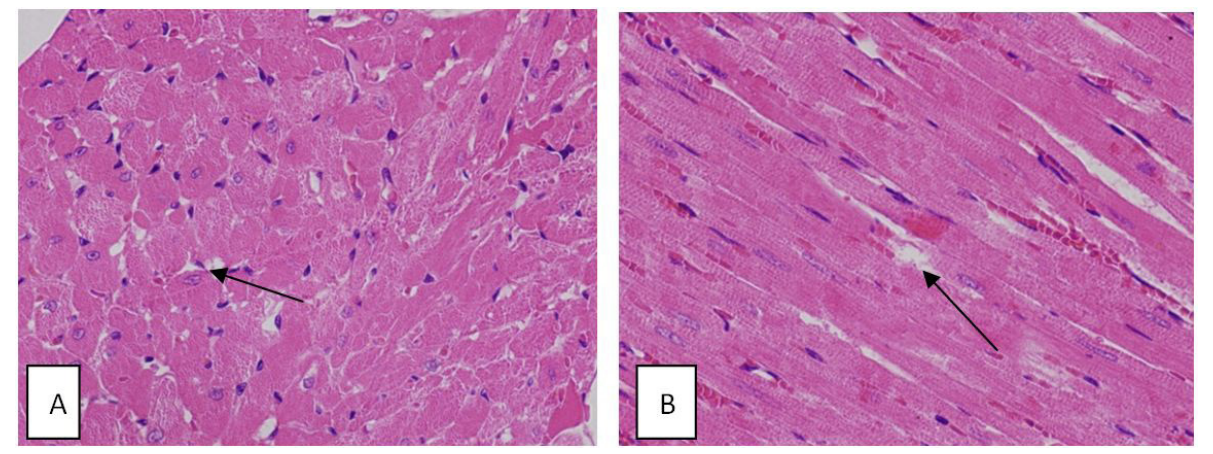

Figure 4: High power microphotographs of rat heart show lipid infiltration compare control with tested group

\section{Discussion}

Hyperlipidemia, a group of metabolic disorders characterized by the elevated levels of lipids, is a major modifiable risk factor for atherosclerosis and cardiovascular disease [5]. These lipids include cholesterol, cholesterol esters, phospholipids, and triglycerides. Increased levels of LDL are related to the development of atherosclerosis $[15,16]$.

The result showed that there were significant increase in hyperlipidemia biomarker which includes TC, TG, LDL and HDL. There were histological changes in organs which confirm the previous result of blood lipid. That mean high fat diet successfully induces hyperlipidemia in rats, to make disease model of hyperlipidemia.

Hyperlipidemia can be the result of an inherited disease in certain breeds of dogs [17]. In pets, hyperlipidemia most often occurs as a consequence of some disorder, hyperlipidemia even can also occur spontaneously after a meal of high-fat diet, particularly table scraps $[9,10]$. Hyperlipidemia is seen most commonly in ponies, miniature horses, and donkeys, and less frequently in standardsize adult horses [18]. In non-ruminants, including primates and man, hyperlipidemia may be increased by dietary manipulations such as feeding excessive cholesterol or fats with high saturated fatty acid content [19].

\section{References}

1. Xu Q-Y, Liu Y-H, Zhang Q, Ma B, Yang Z-D, et al. (2014) Metabolomic analysis of simvastatin and fenofibrate intervention in high-lipid diet-induced hyperlipidemia rats. Acta Pharmacologica Sinica 35: 1265.

2. Watson T, Barrie J (1993) Lipoprotein metabolism and hyperlipidaemia in the clog and cat: A review. J Small Anim Pract 34: 479-87.

3. Ford RB (1996) Clinical management of lipemic patients. The Compendium on continuing education for the practicing veterinarian (USA).

4. Johnson MC (2005) Hyperlipidemia disorders in dogs. Compendium 27: 361-70.

5. Bauer J (1995) Evaluation and dietary considerations in idiopathic hyperlipidemia in dogs. J Am Vet Med Assoc 206: 1684-8.

6. Burtis CA, Ashwood ER (1999) Lipids, lipoproteins and apolipoproteins In: Tietz textbook of clinical chemistry (3 $3^{\text {rd }}$ Edn) Philadelphia: WB Saunders Company, USA.

7. Ginsberg HN (1998) Lipoprotein physiology. Endocrinology and Metabolism Clinics 27: 503-19.

8. Mahley RW, Weisgraber KH, Innerarity T (1974) Canine lipoproteins and atherosclerosis: II. Characterization of the plasma lipoproteins associated with atherogenic and nonatherogenic hyperlipidemia. Circ Res 35: 722-33.

9. Whitney MS (1992) Evaluation of hyperlipidemias in dogs and cats. Semin Vet Med Surg (Small Anim) 7: 292-300.

10. Bauer JE (2004) Lipoprotein-mediated transport of dietary and synthesized lipids and lipid abnormalities of dogs and cats. J Am Vet Med Assoc 224: 668-75. 
11. Bauer JE (1996) Comparative lipid and lipoprotein metabolism. Vet Clin Pathol 25: 49-56.

12. Lee HS, Nam Y, Chung YH, Kim HR, Park ES, et al. (2014) Beneficial effects of phosphatidylcholine on high-fat diet-induced obesity, hyperlipidemia and fatty liver in mice. Life Sci 118: 7-14.

13. Al-Awadi JHH, Rashid KH, Hassen AJ (2013) High Fat Diet Induce Hyperlipidemia Incidences With Sever Changes in Liver Tissue of Male Albino Rats: A Histological and Biochemical Study. Kerbala J Pharmaceutical Sci 6: 21-32.

14. Bancroft J, Stevens A (1990) Theory and Practice of Histological Techniques (3rd Edn) Churchill Livingston. Edinburgh, Scotland.

15. Smith SC, Jackson R, Pearson TA, Fuster V, Yusuf S, et al. (2004) Principles for national and regional guidelines on cardiovascular disease prevention: a scientific statement from the World Heart and Stroke Forum. Circulation 109: 3112-21.

16. Jacobson MS (1998) Heart healthy diets for all children: no longer controversial. J Pediatr 133: 1-2.

17. Xenoulis PG, Steiner JM (2010) Lipid metabolism and hyperlipidemia in dogs. Vet J 183: 12-21.

18. Durham A (2006) Clinical application of parenteral nutrition in the treatment of five ponies and one donkey with hyperlipaemia. Vet Rec 158: 159-64.

19. Nestel P, Poyser A, Hood R, Mills S, Willis M, et al. (1978) The effect of dietary fat supplements on cholesterol metabolism in ruminants. J Lipid Res 19: 899-909.

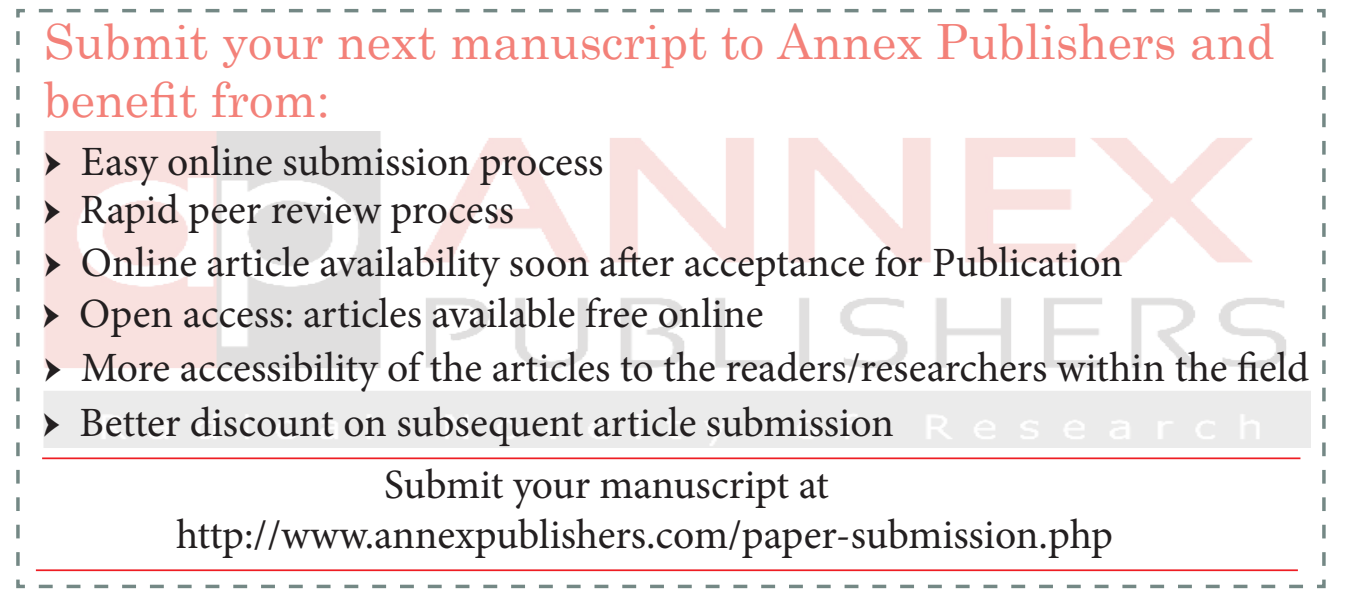

